\title{
A conceptual emotional recognition framework: stress and anger analysis for car accidents
}

\begin{abstract}
In year 2013, 777,817 out of 22,702,211 of registered vehicles were involved m road traffic crashes, with a damage cost of more than 9.3 billion ringgits in Malaysia. Automotive makers have deployed a wide range of active safety systems (traction control, auto cruise and braking, collision avoidance, and lane switching) in recent years. However, these systems failed to significantly reduce road crash statistics. The automotive network comprises the vehicle road and driver. Huge effort had been placed on the vehicle and road, whereas the dnver remains the feeblest part. Consequently, automotive makers should focus their attention on monitoring drivers' emotion, which influences their driving behaviour. This study aims to explore available technology that monitors driver behaviour, besides proposing accident-stimulating emotion that affects driving. Furthermore, a conceptual framework for recognising anger and stress emotion, which is a cause of road crash, is proposed.
\end{abstract}

Keyword: Accident anger; Behaviour; Conceptual; Emotion; Fatigue; Framework; Recognition; Stress; Vehicle 